\title{
AVALIAÇÃO DOS COMPORTAMENTOS DE AVES POEDEIRAS UTILIZANDO SEQÜÊNCIA DE IMAGENS ${ }^{1}$
}

\author{
JOSÉ A. D. BARBOSA FILHO², IRAN J. O. SILVA ${ }^{3}$, MARCO A. N. SILVA ${ }^{4}$, \\ CAIO J. M. SILVA
}

\begin{abstract}
RESUMO: A grande preocupação com o uso de gaiolas dá-se quanto ao espaço oferecido às aves poedeiras, o qual, certamente, afeta seu bem-estar. Sendo assim, este trabalho teve o objetivo de avaliar aspectos relacionados ao comportamento e bem-estar animal, comparando o sistema de criação alternativo (com cama e ninho) com o sistema convencional em gaiolas. Para isso, dois grupos de 20 aves (10 Hy-Line W36 e 10 Hy-Line Brown) em início de produção foram divididos em dois sistemas de criação (cama+ninho e gaiolas) e colocadas em câmara climática: um grupo a $26{ }^{\circ} \mathrm{C}$ e $60 \%$ UR (condição de conforto térmico), e outro a $35{ }^{\circ} \mathrm{C}$ e $70 \%$ UR (condição de estresse térmico), onde eram constantemente monitoradas por câmeras de vídeo. Com base na análise dos comportamentos, verificou-se que o sistema de criação em cama propiciou a expressão de todos os comportamentos naturais relacionados ao conforto das aves, evidenciando o estado de bem-estar das mesmas. Para o sistema em gaiolas, pôde ser constatado que as aves tentavam expressar seus comportamentos naturais, mesmo não tendo condições para isso. Essa impossibilidade de expressão dos comportamentos agravou as condições de estresse provocadas por esse sistema de criação e pela condição ambiental a que as aves foram submetidas.
\end{abstract}

PALAVRAS-CHAVE: bem-estar animal, comportamento animal, análise de imagens.

\section{BEHAVIOR EVALUATION OF LAYING HENS USING IMAGE SEQUENCES}

\begin{abstract}
The concern about the use of cages is related to barren environment and the minimal space offered to the laying hens, which negatively affects bird welfare. The present study focused on the aspect evaluation related to the behavior and welfare of birds, making a comparison between the alternative housing system (with litter and nests) and the conventional cage system. Two groups of 20 laying hens (10 Hy-Line W36 and 10 Hy-line Brown) at the beginning of the production were divided in two breeding systems (litter+nest and cages). Both of them were kept in climatic controlled chambers: one group with an ambient temperature of $26{ }^{\circ} \mathrm{C}$ and $60 \% \mathrm{RH}$ (Thermal Comfort Condition) and the other in a temperature of $35{ }^{\circ} \mathrm{C}$ and $70 \% \mathrm{RH}$ (Heat Thermal Stress Condition). A system of video cameras recorded the behavior of the laying hens. According to the behavior analysis, it was verified that the housing system on litter led to the expression of all natural behaviors related to bird comfort, which permitted to improve welfare conditions. In conventional system (laying cages) it was verified that birds tried to show their natural behavior in spite of lacking condition for it. This impossibility for expressing natural behaviors may have increased stress caused by the housing system and ambient condition.
\end{abstract}

KEYWORDS: poultry welfare, animal behavior, image analysis.

\footnotetext{
${ }^{1}$ Extraído da dissertação do primeiro autor.

${ }^{2}$ Eng $^{\circ}$ Agrícola, Doutorando do curso de Física do Ambiente Agrícola, Departamento de Engenharia Rural, NUPEA/ESALQ/USP, Av. Pádua Dias, 11, Caixa Postal 9, Fone: (OXX19) 3429-4217, Ramal 240, Piracicaba - SP, joseadbf@esalq.usp.br

${ }^{3}$ Eng $^{\mathrm{o}}$ Agrí́cola, Prof. Doutor, Departamento de Engenharia Rural, NUPEA/ESALQ/USP, Piracicaba - SP.

${ }^{4}$ Eng ${ }^{\mathrm{o}}$ Agrônomo, Departamento de Genética. NUPEA/ESALQ/USP, Piracicaba - SP.

${ }^{5}$ Biólogo, Departamento de Genética, NUPEA/ESALQ/USP, Piracicaba - SP.

Recebido pelo Conselho Editorial em: 6-3-2006

Aprovado pelo Conselho Editorial em: 20-12-2006
} 


\section{INTRODUÇÃO}

As limitações impostas pela legislação internacional de bem-estar de aves poedeiras, com relação ao atual sistema de criação (bateria de gaiolas) e inclusive a substituição desse, traz consigo um conjunto de mudanças nas instalações e no manejo das aves, visando à melhor adaptação aos novos requisitos do mercado. Dessas mudanças, que já vêm ocorrendo em alguns países, surgem os sistemas alternativos para a criação de aves poedeiras, tais como o sistema chamado de "gaiolas enriquecidas", e os sistemas de semiconfinamento, que poderão proporcionar às aves uma área com cama e poleiro, além de disponibilizar ninho para a postura.

Grande parte do padrão de comportamento normal da ave é frustrado pelo engaiolamento. $\mathrm{O}$ comportamento de acasalamento, incubação e cuidado com os pintinhos é impedido, e a única compulsão reprodutiva permitida é a de pôr ovos. Elas não podem voar, ciscar, empoleirar-se nem andar livremente. É difícil para a ave limpar suas penas e é impossível "sujar-se" com terra (SINGER, 1991).

A comparação de estudos de comportamento de aves selvagens e domesticadas em ambientes controlados pelo homem indica que o repertório comportamental das aves em ambientes não-confinados, em geral, é preservado, havendo mudanças na freqüência e na intensidade das características comportamentais (CRAIG, 1992).

Sendo assim, o estudo do comportamento animal torna-se uma importante ferramenta para a avaliação dos sistemas de criação, além de fornecer muitas respostas a questões básicas da etologia. Dentre as ferramentas utilizadas para o estudo dos comportamentos dos animais, a análise de imagens tem-se destacado. Segundo XIN et al. (1998), um sistema para a análise de imagens deverá ser constituído por uma microcâmera, uma placa de captura de imagem instalada em computador e um programa que execute a aquisição, o processamento e a classificação das imagens dos animais.

O desconforto térmico em aves de postura também provoca uma série de consequiências que, por sua vez, estão intimamente ligadas à queda no consumo de ração, menor taxa de crescimento, maior consumo de água, aceleração do ritmo cardíaco, alteração da conversão alimentar, queda na produção de ovos e maior incidência de ovos com casca mole (PAYNE, 1967).

Sendo assim, este trabalho teve como objetivo avaliar os aspectos relacionados ao comportamento animal, principalmente quanto à presença de cama ou de ninho em substituição ao sistema convencional (gaiolas), utilizando seqüências de imagens das aves.

\section{MATERIAL E MÉTODOS}

A pesquisa foi realizada em câmara climática nas dependências do Núcleo de Pesquisa em Ambiência (NUPEA), junto ao Departamento de Engenharia Rural da ESALQ/USP.

Um total de 20 aves de cada linhagem (Hy-Line W36 de Hy-Line Brown), com 21 semanas, foi separado em dois tipos de sistemas de criação, sendo, respectivamente, dez aves colocadas no sistema de criação em gaiola e dez aves no sistema cama+ninho, conforme os seguintes tratamentos:

Tratamento C1 - Sistema de criação com cama e ninho, e

Tratamento C2 - Sistema de criação em gaiolas.

Todas as aves foram expostas a cada uma das condições ambientais (A1 e A2) durante 14 dias consecutivos, em ambiente controlado, de acordo com SEVEGNANI et al. (2001). Porém, com período de adaptação gradativa ao ambiente da câmara climática, totalizando 21 dias de exposição ao ambiente controlado, conforme os tratamentos abaixo:

Tratamento A1 - Exposição ao ambiente com temperatura de $26{ }^{\circ} \mathrm{C} \pm 2{ }^{\circ} \mathrm{C}$ e $60 \% \pm 2 \%$ de umidade relativa (condição de conforto térmico), e 
Tratamento A2 - Exposição ambiente com temperatura de $35{ }^{\circ} \mathrm{C} \pm 2{ }^{\circ} \mathrm{C}$ e $70 \% \pm 2 \%$ de umidade relativa (condição de estresse térmico).

\section{Classificação do ambiente térmico}

Os valores de temperatura de bulbo seco (Tbs, ${ }^{\circ} \mathrm{C}$ ) e umidade relativa (UR, \%) foram coletados no interior da câmara climática por meio de miniestações meteorológicas e "datalogger" $\mathrm{HOBO}^{\circledR}$. As miniestações meteorológicas foram instaladas, respectivamente, a 1,70; 1,50 e a 0,50 $\mathrm{m}$ de altura em relação ao piso da câmara, sendo as leituras realizadas a cada 15 min ao longo das 24 h, o que possibilitou traçar um perfil de temperatura dentro da câmara climática.

Para caracterizar a zona de conforto térmico adequada às aves, foi determinado o índice de conforto térmico entalpia, por meio da fórmula descrita por VILLA NOVA (1999), citado por FURLAN (2001), em que:

$$
\mathrm{H}=6,7+0,243 \mathrm{Tbs}+\left\{\frac{\mathrm{UR}}{100} 10^{\frac{7,5 \mathrm{Tbs}}{237,3 \mathrm{~T} \mathrm{Tbs}}}\right\}
$$

em que,

$\mathrm{H}$ - entalpia, $\mathrm{kcal} \mathrm{kg}^{-1}$ ar seco;

Tbs - temperatura do bulbo seco, ${ }^{\circ} \mathrm{C}$, e

UR - umidade relativa do ar, $\%$.

\section{Seqüência de imagens}

As imagens foram registradas por câmeras de vídeo instaladas no teto da câmara climática que, por sua vez, eram ligadas a um microcomputador equipado com placa de captura de imagens e "software" para a análise. Todas as aves foram identificadas por meio de sistema de marcação individual, com pintura do dorso com tinta não-tóxica (RUDKIN \& STEWART, 2003), o que possibilitou o acompanhamento e a análise dos comportamentos de cada ave.

As imagens foram registradas a cada segundo, por uma hora, na parte da manhã (das 10 às $11 \mathrm{~h}$ ) e duas horas na parte da tarde (das 13 às $14 \mathrm{~h}$ e das 16 às $17 \mathrm{~h}$ ), durante dois dias consecutivos, para cada sistema de criação e condição ambiental proposta. As imagens, gravadas a cada segundo, foram gerenciadas pelo "software" VIDEOCAP 5.1 ${ }^{\circledR}$, sendo armazenadas em banco de dados. Durante o período da noite, não foram registrados dados de comportamento.

Após as análises, foram calculadas as percentagens médias de tempo de expressão de cada comportamento, bem como o período, em minutos e segundos, de duração de cada comportamento.

\section{Parâmetros comportamentais}

Os padrões comportamentais foram avaliados de acordo com RUDKIN \& STEWART (2003), levando-se em consideração as atividades desenvolvidas por cada ave.

Foram avaliados os seguintes padrões comportamentais:

a) Comendo - Quando a ave está se alimentando, comportamento caracterizado quando a ave se encontrava com a cabeça no comedouro;

b) Bebendo - Quando a ave está bebendo água, caracterizado quando a ave estava bicando o bebedouro tipo Nipple;

c) Investigando penas - Comportamento não-agressivo, caracterizado quando a ave investigava suas próprias penas com o bico ou investigava as penas de outras aves;

d) Banho de areia-Comportamento característico das aves, que envolve em sua caracterização uma seqüência de ciscar e jogar "areia" sobre seu corpo, além de movimentos rápidos de chacoalhar as penas; 
e) Movimentos de conforto - São considerados comportamentos apresentados pelas aves quando essas se encontravam em condições de conforto e bem-estar; são considerados como movimentos de conforto comportamentos como: bater e esticar as asas e chacoalhar as penas;

f) Ciscando - Outro comportamento considerado como característico das aves, caracterizado quando a ave explora seu território com seus pés e bico;

g) Empoleirar - Comportamento associado ao bem-estar das aves; por ser um comportamento natural, é caracterizado quando a ave sobe em algum aparato que se encontra acima do nível do solo;

h) Agressividade - Comportamento relacionado à condição de se estabelecer dominância no grupo ou a condições de estresse, sendo geralmente caracterizado por bicadas rápidas e fortes em locais como a crista e outras partes da cabeça;

i) Sentada - Comportamento caracterizado quando a ave senta na cama ou substrato onde se encontra;

j) Procurando ninho - Comportamento considerado como de pré-postura; é detectável e caracterizado como entradas rápidas e freqüentes ao ninho, como tentativa de avaliar o local antes da postura dos ovos;

k) Postura - Caracterizado como a presença da ave sentada no ninho e com a comprovação da presença do ovo, e

1) Parada - Comportamento caracterizado quando a ave não apresenta nenhum movimento ou, aparentemente, não se enquadra em nenhum dos comportamentos anteriores.

\section{RESULTADOS E DISCUSSÃO}

\section{Classificação do ambiente térmico}

Na Figura 1, mostra-se o comportamento da entalpia ( $\mathrm{kJ} \mathrm{kg}^{-1}$ ar seco) para as condições de conforto e estresse térmico, bem como a respectiva faixa com os valores-limites considerados como "ideais" para aves.

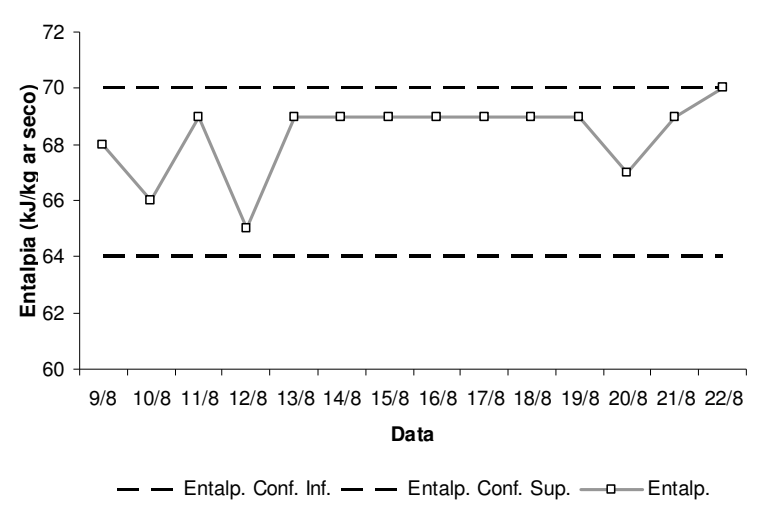

A

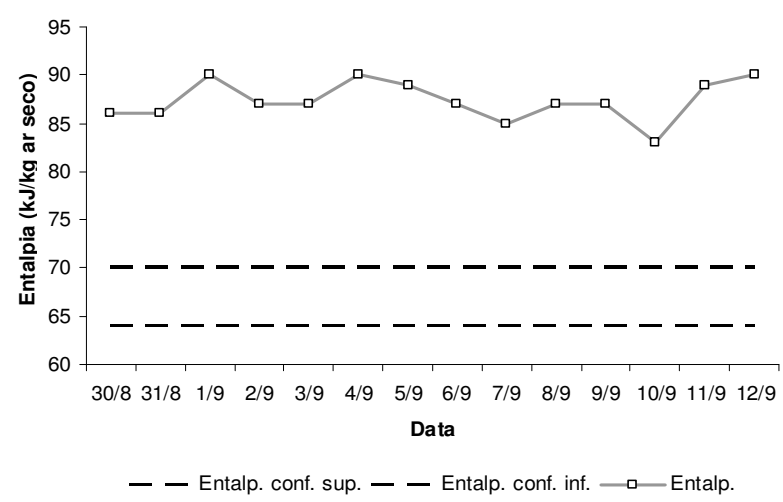

B

FIGURA 1. Valores de entalpia em câmara climática para a condição de conforto (A) e estresse (B), com os respectivos limites: inferior e superior de conforto.

A diferença entre as condições estudadas pode ser visualizada analisando-se a Figura 1, em que, na situação A, nota-se que os valores médios de quantidade de energia existente na massa de ar seco estão dentro das faixas limites de conforto para o índice; por sua vez, analisando-se a situação $\mathrm{B}$, nota-se que esse mesmo fato não é observado, quando a análise é realizada para a condição de estresse, em que os valores da entalpia são superiores aos limites de conforto do índice, caracterizando a condição de estresse térmico. 


\section{Parâmetros comportamentais}

\section{Expressão dos comportamentos para o sistema de criação em cama}

\section{Condição de conforto térmico}

Pela Tabela 1, verifica-se, na comparação entre linhagens, que a linhagem Hy-Line Brown, no período da manhã, permaneceu maior percentagem de tempo comendo, bebendo, ciscando, procurando ninho, tomando banho de areia e investigando penas, comparada com a linhagem HyLine W36. Em contrapartida, a linhagem Hy-Line W36 passou maior percentagem de tempo empoleirada e em postura.

TABELA 1. Valores percentuais de tempo médio, em que as aves expressaram seus comportamentos na condição de conforto térmico.

\begin{tabular}{|c|c|c|c|c|c|c|}
\hline \multicolumn{7}{|c|}{ Condição de Conforto } \\
\hline \multirow[b]{2}{*}{$\begin{array}{c}\text { Padrão de } \\
\text { comportamentos }\end{array}$} & \multicolumn{2}{|c|}{ Período da manhã } & \multicolumn{2}{|c|}{ Período da tarde } & \multicolumn{2}{|c|}{ Total } \\
\hline & $\begin{array}{l}\text { Hy-Line } \\
\text { Brown }\end{array}$ & $\begin{array}{l}\text { Hy-Line } \\
\text { W36 }\end{array}$ & $\begin{array}{l}\text { Hy-Line } \\
\text { Brown }\end{array}$ & $\begin{array}{l}\text { Hy-Line } \\
\text { W36 }\end{array}$ & $\begin{array}{l}\text { Hy-Line } \\
\text { Brown }\end{array}$ & $\begin{array}{l}\text { Hy-Line } \\
\text { W36 }\end{array}$ \\
\hline Comendo & 32 & 28 & 23 & 20 & 27,5 & 24 \\
\hline Bebendo & 4 & 2 & 4 & 1 & 4 & 1,5 \\
\hline Ciscando & 29 & 11 & 23 & 14 & 26 & 12,5 \\
\hline Proc. Ninho & 21 & 13 & 12 & 0,4 & 16,5 & 6,7 \\
\hline Postura & - & 26 & - & 18 & - & 22 \\
\hline Mov. conforto & 1 & 1 & 1 & 1 & 1 & 1 \\
\hline Invest. penas & 6 & 4 & 7 & 7 & 6,5 & 5,5 \\
\hline Empoleirar & - & 15 & 9 & 19 & 4,5 & 17 \\
\hline Banho de areia & 7 & - & 9 & 4 & 8 & 2 \\
\hline Agressividade & - & - & - & 1 & - & 0,5 \\
\hline Sentada & - & - & 8 & 12 & 4 & 6 \\
\hline Parada & - & - & 4 & 3 & 2 & 1,5 \\
\hline Total & 100 & 100 & 100 & 100,4 & 100 & 100,2 \\
\hline
\end{tabular}

O fato de aparecerem valores de percentagem maiores que $100 \%$ na tabela aconteceu devido a momentos com ocorrência de comportamentos acontecendo simultaneamente para uma mesma ave, tais como: empoleirar e investigar penas.

Para o período da tarde, a diferença ocorreu devido à expressão de comportamentos como o de sentar, ficar parada e a ocorrência de agressividade. Outro ponto interessante é a verificação do comportamento de postura na parte da tarde que, como ocorrido na parte da manhã, só foi constatado pela linhagem Hy-Line W36.

$\mathrm{Na}$ avaliação dos comportamentos para a condição de conforto e sistema de criação "cama+ninho", foi evidente, também, a expressão dos chamados "comportamentos de conforto" por parte das aves.

Um fator que exerce muita influência na expressão dos comportamentos das aves é o horário, uma vez que todos os animais seguem um biorritmo. A esse biorritmo está ligado principalmente o aspecto do fotoperíodo que, neste trabalho, foi de 16 horas de luz e 8 horas de escuro, sendo o período de luz dentro da câmara compreendido entre o horário das 8 às 24 h.

Isso explica a influência do período de avaliação (manhã e tarde) na expressão dos comportamentos, ou seja, no período da manhã, as aves passaram mais tempo comendo, certamente devido ao horário de arraçoamento.

Outros comportamentos que também merecem destaque no período da manhã, são os comportamentos de ciscar e de procurar o ninho, além do comportamento de empoleirar, que aparece com percentagem de tempo (15\%) considerável para a linhagem Hy-Line W36. 
Para o período da tarde, nota-se ligeira queda no comportamento de comer e aumento nos comportamentos de investigar penas, empoleirar, tomar banho de areia e ficar parada; além desses comportamentos, aparece também o comportamento de agressividade.

\section{Condição de estresse térmico}

Com relação às linhagens estudadas, pôde-se observar que a linhagem Hy-Line W36 passou mais tempo comendo do que a linhagem Hy-Line Brown, tanto para o período da manhã quanto para o período da tarde. Outro ponto importante a ser observado é quanto à percentagem de tempo que as aves da linhagem Hy-Line Brown passaram paradas (24\%) com relação às da linhagem HyLine W36 (12\%), o que evidencia que essa linhagem é menos resistente às condições de estresse térmico.

Com relação aos comportamentos característicos de conforto, tais como, banho de areia e movimentos de conforto, esses não ocorreram para a condição de estresse, o que confirma os efeitos do estresse na expressão dos comportamentos normais das aves.

TABELA 2. Valores percentuais de tempo médio, em que as aves expressaram seus comportamentos na condição de estresse térmico.

\begin{tabular}{|c|c|c|c|c|c|c|}
\hline \multicolumn{7}{|c|}{ Condição de Estresse } \\
\hline \multirow[b]{2}{*}{$\begin{array}{c}\text { Padrão de } \\
\text { comportamentos }\end{array}$} & \multicolumn{2}{|c|}{ Período da manhã } & \multicolumn{2}{|c|}{ Período da tarde } & \multicolumn{2}{|c|}{ Total } \\
\hline & $\begin{array}{l}\text { Hy-Line } \\
\text { Brown }\end{array}$ & $\begin{array}{l}\text { Hy-Line } \\
\text { W36 }\end{array}$ & $\begin{array}{l}\text { Hy-Line } \\
\text { Brown }\end{array}$ & $\begin{array}{l}\text { Hy-Line } \\
\text { W36 }\end{array}$ & $\begin{array}{l}\text { Hy-Line } \\
\text { Brown }\end{array}$ & $\begin{array}{l}\text { Hy-Line } \\
\text { W36 }\end{array}$ \\
\hline Comendo & 20 & 36 & 16 & 25 & 18 & 30,5 \\
\hline Bebendo & 8 & 9 & 6 & 6 & 7 & 7,5 \\
\hline Ciscando & 19 & 25 & 26 & 19 & 22,5 & 22 \\
\hline Proc. Ninho & - & - & - & 25 & - & 12,5 \\
\hline Postura & - & - & - & - & - & - \\
\hline Mov. conforto & - & - & - & - & - & - \\
\hline Invest. penas & 3 & 4 & 5 & 5 & 4 & 4,5 \\
\hline Empoleirar & 24 & - & 19 & 4 & 21,5 & 2 \\
\hline Banho de areia & - & - & - & - & - & - \\
\hline Agressividade & - & - & - & - & - & - \\
\hline Sentada & 2 & 14 & 4 & 6 & 3 & 10 \\
\hline Parada & 24 & 12 & 24 & 10 & 24 & 11 \\
\hline Total & 100 & 100 & 100 & 100 & 100 & 100 \\
\hline
\end{tabular}

Com relação à expressão dos comportamentos das aves na condição de estresse térmico em comparação com a condição de conforto, as aves quase não apresentam a expressão dos chamados "movimentos de conforto"; outra característica da situação de estresse apresentada pelas aves é o aumento no comportamento de beber água, concordando com STURKIE (1967), que foi de 75\% para Hy-Line Brown e de surpreendentes 400\% para Hy-Line W36.

De acordo com PAYNE (1967), sob condições de estresse térmico, ocorrerá também queda brusca no comportamento de se alimentar que, neste caso, só pôde ser verificado para a linhagem Hy-Line Brown (53\%).

Um fato que também chama a atenção é o grande aumento na expressão dos comportamentos de sentar e de ficar parada (até 12 vezes maior, no caso da Hy-Line Brown), que certamente são medidas comportamentais que influenciam diretamente na fisiologia dos animais, de modo a não aumentar a produção de calor através de movimentos.

\section{Expressão dos comportamentos para o sistema de criação em gaiolas}

A análise dos comportamentos para o sistema convencional de criação, ou seja, para o sistema em gaiolas, foi mais difícil de ser feita, uma vez que problemas como o posicionamento 
das câmeras de vídeo (instaladas no teto da câmara climática) e a quantidade de aves por gaiola (5 aves por gaiola) impediram análise mais apurada das imagens, como a feita para a condição de "cama+ninho".

Diante disso, as análises foram feitas para o conjunto das aves, ou para o conjunto de seus comportamentos nas condições ambientais propostas. Sendo assim, para a condição de conforto térmico, pôde ser observado que o grupo de aves da linhagem Hy-Line Brown e da Hy-Line W36, para os períodos da manhã e da tarde, passaram a maior parte do tempo comendo; outros comportamentos que também puderam ser evidenciados, foram o de investigar penas e o de beber água.

Para a condição de estresse térmico, os comportamentos detectados com maior frequiência foram os de sentar-se, ficar parada e o de beber água, todos característicos de aves em condição de confinamento e sob condições de estresse térmico. O comportamento de comer também apresentou grande redução (cerca de 50\%), principalmente para a linhagem Hy-Line Brown. Comportamentos indicativos de conforto e bem-estar não foram detectados, uma vez que as dimensões das gaiolas e as condições ambientais não possibilitaram a expressão dos mesmos.

\section{CONCLUSÕES}

Pela análise dos comportamentos das aves, pôde ser verificada a ocorrência dos comportamentos naturais e de conforto no sistema de criação em cama, que certamente possibilitou melhores condições de bem-estar para as aves, comparado com o sistema de criação em gaiolas, onde a expressão desses comportamentos praticamente não ocorreu. Sob condições de estresse térmico, o sistema de criação em cama foi o que se mostrou mais adequado sob o ponto de vista de redução dos efeitos do estresse causados pelos altos valores das variáveis ambientais.

\section{REFERÊNCIAS}

CRAIG, J.V. Measuring social behavior in poultry. Poultry Science, Savoy, v.71, n.4, p. 650-7, 1992.

FURLAN, R.A. Avaliação da nebulização e abertura de cortinas na redução da temperatura do ar em ambiente protegido. 2001. 146 f. Tese (Doutorado em Irrigação e Drenagem) - Escola Superior de Agricultura "Luiz de Queiroz”, Universidade de São Paulo, Piracicaba, 2001.

PAYNE, G.C. Environmental temperature and egg production: the physiology of the domestic fowl. Edinburgh: Horton-Smith, 1967. p.235-41.

RUDKIN, C.; STEWART, G.D. Behaviour of hens in cages - A pilot study using video tapes. A Report for the Rural Industries Research and Development Corporation (RIRDC), Queensland, v.40, n.477, p.102, 2003.

SEVEGNANI, K.B.; MACARI, M.; NÄÄS, I.A.; MOURA, D.J.; SILVA, I.J.O. Variação da temperatura média corporal de frangos de corte em terminação, submetidos às temperaturas de $24 \mathrm{e}$ $32{ }^{\circ} \mathrm{C}$, em câmara climática. Revista Brasileira de Ciência Avícola, Campinas, supl. 3, p.58, 2001.

SINGER, P. Animal liberation. Rev. ed. New York: Harper Perennial - Avon Books, 1991. 320 p.

STURKIE, P.D. Fisiologia aviaria. Zaragoza: Ed. Acribia, 1967. 607 p.

XIN, J.; BECK, H.; HALSEY, L.; FLETCHER, J.; ZAZUETA, F. Using digital cameras and the internet to identify plant insect and disease problems. In: INTERNATIONAL CONFERENCE ON COMPUTERS IN AGRICULTURE, 17., 1998. Orlando. Proceedings... Orlando: ASAE, 1998. p.327-9. 